\title{
$q$-Extension of a Multivariable and Multiparameter Generalization of the Gottlieb Polynomials in Several Variables
}

\author{
Junesang CHOI and H. M. SRIVASTAVA \\ Dongguk University and University of Victoria \\ (Communicated by K. Shinoda)
}

\begin{abstract}
While considering some families of polynomials which are orthogonal on a finite or enumerable set of points, Gottlieb was led in the year 1938 to what are now popularly known as the Gottlieb polynomials $\varphi_{n}(x ; \lambda)$. This one-parameter family of polynomials has ever since then been cited widely and investigated extensively in several books, monographs and journal articles. In the present sequel to some of the aforementioned investigations, we introduce and systematically investigate a basic (or $q$-) extension of a multivariable and multiparameter generalization of the Gottlieb polynomials $\varphi_{n}(x ; \lambda)$. We also establish a set of three new families of generating functions for the generalized $q$-Gottlieb polynomials defined here.
\end{abstract}

\section{Introduction and Preliminaries}

Generating functions play an important rôle in the investigation of various useful properties of the sequences which they generate. They are used in finding many properties, characteristics and formulas for the generated numbers and polynomials in a wide variety of research subjects in (for example) modern combinatorics. For a systematic introduction to, and for several interesting (and useful) applications of the various methods of obtaining linear, bilinear, bilateral or mixed multilateral generating functions for a fairly wide variety of sequences of special functions (and polynomials) in one, two and more variables, among much abundant available literature, we refer the interested reader to the extensive work presented in the monograph on this subject by Srivastava and Manocha [23]. While considering some orthogonal polynomials on a finite or enumerable set of points, Gottlieb [7] developed and studied the following interesting oneparameter family of polynomials (see also [3], [8], [9], [10], [14, p. 303] and [23, pp. 185186]):

Received January 30, 2013

2010 Mathematics Subject Classification: 33C65, 33C99 (Primary), 33C05, 33C20 (Secondary)

Key words and phrases: Generating and basic (or $q$-) generating functions, Generalized hypergeometric function ${ }_{r} F_{S}$ and the generalized basic (or $q$-) hypergeometric function ${ }_{r} \Phi_{S}$, Orthogonality on a finite or enumerable set of points, Gottlieb polynomials and the generalized $q$-Gottlieb polynomials, Jacobi and Meixner polynomials, Lauricella's multiple hypergeometric series, $q$-Binomial theorem and Ramanujan's ${ }_{1} \Psi_{1}$-sum, Srivastava's general basic (or $q$-) hypergeometric series in several variables 


$$
\begin{aligned}
\varphi_{n}(x ; \lambda): & =e^{-n \lambda} \sum_{k=0}^{n}\left(\begin{array}{l}
n \\
k
\end{array}\right)\left(\begin{array}{l}
x \\
k
\end{array}\right)\left(1-e^{\lambda}\right)^{k} \\
& =e^{-n \lambda}{ }_{2} F_{1}\left(-n,-x ; 1 ; 1-e^{\lambda}\right),
\end{aligned}
$$

where ${ }_{2} F_{1}$ denotes Gauss's hypergeometric series which is the case $r-1=s=1$ of the generalized hypergeometric series ${ }_{r} F_{S}$ with $r$ numerator and $s$ denominator parameters $(r, s \in$ $\left.\mathbf{N}_{0}:=\mathbf{N} \cup\{0\}\right)$ defined by

$$
\begin{aligned}
&{ }_{r} F_{s}\left[\begin{array}{c}
\alpha_{1}, \ldots, \alpha_{r} ; \\
z \\
\beta_{1}, \ldots, \beta_{s} ;
\end{array}\right]:=\sum_{n=0}^{\infty} \frac{\left(\alpha_{1}\right)_{n} \cdots\left(\alpha_{r}\right)_{n}}{\left(\beta_{1}\right)_{n} \cdots\left(\beta_{s}\right)_{n}} \frac{z^{n}}{n !} \\
&={ }_{r} F_{S}\left(\alpha_{1}, \ldots, \alpha_{r} ; \beta_{1}, \ldots, \beta_{s} ; z\right),
\end{aligned}
$$

$\mathbf{N}$ being the set of positive integers. Here $(\lambda)_{v} \quad(\lambda, v \in \mathbf{C})$ denotes the Pochhammer symbol (or the shifted factorial) which is defined, in terms of the familiar Gamma function, by

$$
(\lambda)_{v}:=\frac{\Gamma(\lambda+v)}{\Gamma(\lambda)}= \begin{cases}1 & (v=0 ; \lambda \in \mathbf{C} \backslash\{0\}) \\ \lambda(\lambda+1) \cdots(\lambda+n-1) & (v=n \in \mathbf{N} ; \lambda \in \mathbf{C}),\end{cases}
$$

it being understood conventionally that $(0)_{0}:=1$ and assumed tacitly that the Gamma quotient exists.

Gottlieb [7] presented many interesting properties and identities for the polynomials $\varphi_{n}(x ; \lambda)$, which incidentally were denoted by $\ell_{n}(x)$ in [7] and by $\ell_{n}(x ; \lambda)$ in [23, p. 185, Problem 47]. Some of these identities include the following two generating functions (see, for details, [12, p. 91], [14, p. 303], [23, pp. 185-186] and [24, p. 37]):

$$
\sum_{n=0}^{\infty} \varphi_{n}(x ; \lambda) t^{n}=(1-t)^{x}\left(1-t e^{-\lambda}\right)^{-x-1} \quad(|t|<1)
$$

and

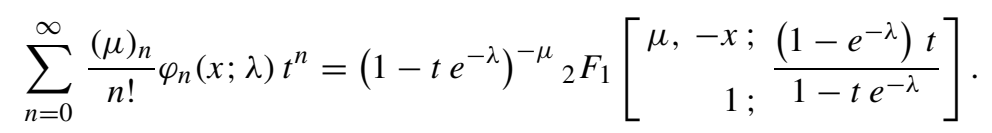


In fact, it is also known that (see, for example, [23, p. 449, Problem 20(i)])

$$
\begin{aligned}
\sum_{n=0}^{\infty}\left(\begin{array}{c}
m+n \\
n
\end{array}\right) \varphi_{m+n}(x ; \lambda) t^{n}=(1-t)^{x-m}\left(1-t e^{-\lambda}\right)^{-x-1} \\
\cdot \varphi_{m}\left(x ; \log \left(\frac{e^{\lambda}-t}{1-t}\right)\right) \quad\left(m \in \mathbf{N}_{0} ;|t|<1\right),
\end{aligned}
$$

which, in the special case when $m=0$, would reduce immediately to the generating function (1.4).

The Gottlieb polynomials $\varphi_{n}(x ; \lambda)$ defined by $(1.1)$ are contained in the classical Jacobi polynomials $P_{n}^{(\alpha, \beta)}(x)$ given by (see, for example, [24, Chapter IV])

$$
\begin{aligned}
P_{n}^{(\alpha, \beta)}(x)=P_{n}^{(\beta, \alpha)}(-x) & :=\sum_{k=0}^{n}\left(\begin{array}{c}
n+\alpha \\
n-k
\end{array}\right)\left(\begin{array}{c}
n+\beta \\
k
\end{array}\right)\left(\frac{x-1}{2}\right)^{k}\left(\frac{x+1}{2}\right)^{n-k} \\
& =\left(\begin{array}{c}
n+\alpha \\
n
\end{array}\right){ }_{2} F_{1}\left(-n, n+\alpha+\beta+1 ; \alpha+1 ; \frac{1-x}{2}\right)
\end{aligned}
$$

as well as in the Meixner polynomials $M_{n}(x ; \beta, c)$ defined by (see [13] and [23, p. 443, Problem 5(i)])

$$
M_{n}(x ; \beta, c):=(\beta)_{n 2} F_{1}\left(-n,-x ; \beta ; 1-\frac{1}{c}\right)=n ! P_{n}^{(\beta-1,-\beta-x-n)}\left(\frac{2}{c}-1\right) .
$$

In fact, we have the following relationships:

$$
\varphi_{n}(x ; \lambda)=\frac{e^{-n \lambda}}{n !} M_{n}\left(x ; 1, e^{\lambda}\right)=e^{-n \lambda} P_{n}^{(0,-1-x-n)}\left(2 e^{\lambda}-1\right) .
$$

We now turn to the basic (or $q$-) shifted factorial $(\lambda ; q)_{\mu}$ which is defined, for $q, \lambda, \mu \in$ C $(|q|<1)$, by (see also [21])

$$
(\lambda ; q)_{\mu}:=\prod_{j=0}^{\infty}\left(\frac{1-\lambda q^{j}}{1-\lambda q^{\mu+j}}\right) \quad(|q|<1 ; \lambda, \mu \in \mathbf{C}),
$$

so that

$$
\begin{gathered}
(\lambda ; q)_{n}:=\left\{\begin{array}{lc}
1 & (n=0) \\
\prod_{j=0}^{n-1}\left(1-\lambda q^{j}\right) & (n \in \mathbf{N}),
\end{array}\right. \\
(\lambda ; q)_{\infty}:=\prod_{j=0}^{\infty}\left(1-\lambda q^{j}\right) \quad(|q|<1 ; \lambda \in \mathbf{C})
\end{gathered}
$$


and

$$
\lim _{q \downarrow 1}\left\{\frac{\left(q^{\lambda} ; q\right)_{n}}{\left(q^{\mu} ; q\right)_{n}}\right\}=\frac{(\lambda)_{n}}{(\mu)_{n}} \quad\left(n \in \mathbf{N}_{0} ; \lambda \in \mathbf{C} ; \mu \in \mathbf{C} \backslash \mathbf{Z}_{0}\right)
$$

in terms of the Pochhammer symbol defined by (1.3), $\mathbf{C}$ being the set of complex numbers and

$$
\mathbf{Z}_{0}^{-}:=\mathbf{Z}^{-} \cup\{0\} \quad\left(\mathbf{Z}^{-}:=\{-1,-2,-3, \ldots\}\right) .
$$

A basic (or $q$-) extension ${ }_{r} \Phi_{s}$ of the generalized hypergeometric series ${ }_{r} F_{S}$ in (1.2) is defined by (see, for example, [6] and [22, p. 347, Eq. 9.4(272)])

$$
\begin{aligned}
{ }_{r} \Phi_{s}\left[\begin{array}{c}
a_{1}, \ldots, a_{r} ; \\
b_{1}, \ldots, b_{s} ;
\end{array}\right] & ={ }_{r} \Phi_{s}\left(a_{1}, \ldots, a_{r} ; b_{1}, \ldots, b_{s} ; q, z\right) \\
& :=\sum_{k=0}^{\infty}(-1)^{(1-r+s) k} q^{(1-r+s)\left(\frac{k}{2}\right)} \frac{\left(a_{1} ; q\right)_{k} \cdots\left(a_{r} ; q\right)_{k}}{\left(b_{1} ; q\right)_{k} \cdots\left(b_{s} ; q\right)_{k}} \frac{z^{k}}{(q ; q)_{k}}
\end{aligned}
$$

provided that the generalized basic (or q-) hypergeometric series in (1.14) converges. We note that, since

$$
\left(q^{-n} ; q\right)_{k}= \begin{cases}(-1)^{k} q^{\left(\begin{array}{l}
k \\
2
\end{array}\right)-n k} \frac{(q ; q)_{n}}{(q ; q)_{n-k}} & \left(n, k \in \mathbf{N}_{0} ; 0 \leqq k \leqq n\right) \\
0 & \left(n, k \in \mathbf{N}_{0} ; n \geqq k+1\right),\end{cases}
$$

the series in (1.14) would terminate and yield a polynomial of degree $n$ in $z$ whenever any of the numerator parameters $a_{1}, \ldots, a_{r}$ is of the form $q^{-n}\left(n \in \mathbf{N}_{0}\right)$.

Basic (or $q$-) hypergeometric series and polynomials are useful in a wide variety of fields including, for example, theory of partitions, number theory, combinatorial analysis, finite vector space, Lie theory, particle physics, non-linear electric circuit theory, mechanical engineering, theory of heat conduction, quantum mechanics, cosmology, and statistics (see [22, pp. 346-351] and the references cited therein).

Recently, Khan and Akhlaq [8] introduced and investigated the Gottlieb polynomials in two and three variables and derived their generating functions. On the other hand, Khan and Asif [9] investigated certain interesting generating functions for a $q$-extension of the Gottlieb polynomials. Subsequently, by suitably modifying method used by Khan and Akhlaq [8], Choi [3] presented a generalization of the Gottlieb polynomials in $m$ variables and derived two generating functions of the $m$-variable Gottlieb polynomials $\varphi_{n}^{m}(\cdot)$. Choi $[4,5]$ has also investigated several generating functions for the $q$-extensions of the two-variable Gottlieb polynomials $\varphi_{n}^{2}(\cdot)$ and the three-variable Gottlieb polynomials $\varphi_{n}^{3}(\cdot)$. The main object of the present sequel to the aforementioned investigations is to introduce and systematically 
investigate a basic (or $q$-) extension of a multivariable and multiparameter generalization of the Gottlieb polynomials $\varphi_{n}(x ; \lambda)$. We also establish a set of three new families of generating functions for the generalized $q$-Gottlieb polynomials defined in this paper. Several other investigations dealing substantially with $q$-series and $q$-polynomials include (for example) the recent works $[1,20,21]$.

In our present investigation, we shall need the $q$-binomial theorem:

$$
{ }_{1} \Phi_{0}(\lambda ;-; q, z):=\sum_{k=0}^{\infty} \frac{(\lambda ; q)_{k}}{(q ; q)_{k}} z^{k}=\frac{(\lambda z ; q)_{\infty}}{(z ; q)_{\infty}} \quad(|q|<1 ;|z|<1),
$$

which is, in fact, the special case $\mu=q$ of the celebrated Ramanujan's ${ }_{1} \Psi_{1}$-sum:

$$
\begin{aligned}
{ }_{1} \Psi_{1}(\lambda ; \mu ; q, z):= & \sum_{k=-\infty}^{\infty} \frac{(\lambda ; q)_{k}}{(\mu ; q)_{k}} z^{k}=\frac{(\lambda z ; q)_{\infty}\left(\frac{q}{\lambda z} ; q\right)_{\infty}(q ; q)_{\infty}\left(\frac{\mu}{\lambda} ; q\right)_{\infty}}{(z ; q)_{\infty}\left(\frac{\mu}{\lambda z} ; q\right)_{\infty}(\mu ; q)_{\infty}\left(\frac{q}{\lambda} ; q\right)_{\infty}} \\
& \left(|q|<1 ;|\lambda|>|q| ;|\mu|<1 ;\left|\frac{\mu}{\lambda}\right|<|z|<1\right),
\end{aligned}
$$

where

$$
(\lambda ; q)_{-n}:=\prod_{j=1}^{n}\left(\frac{1}{1-\lambda q^{-j}}\right)=\frac{1}{\left(\lambda q^{-n} ; q\right)_{n}}=\frac{\left(-\frac{q}{\lambda}\right)^{n} q^{\left(\begin{array}{c}
n \\
2
\end{array}\right)}}{\left(\frac{q}{\lambda} ; q\right)_{n}} \quad\left(n \in \mathbf{N}_{0}\right) .
$$

\section{2. $q$-Extensions of the Gottlieb Polynomials in One and More Variables}

We begin by recalling the definition of a multivariable and multiparameter generalization of the Gottlieb polynomials $\varphi_{n}(x ; \lambda)$ as Definition 1 below.

Definition 1 (see Choi [3]). The Gottlieb polynomials $\varphi_{n}^{m}\left(x_{1}, \ldots, x_{m} ; \lambda_{1}, \ldots, \lambda_{m}\right)$ in $m$ variables $x_{1}, \ldots, x_{m}$ and with $m$ parameters $\lambda_{1}, \ldots, \lambda_{m}$ is defined by

$$
\begin{gathered}
\varphi_{n}^{m}\left(x_{1}, \ldots, x_{m} ; \lambda_{1}, \ldots, \lambda_{m}\right) \\
:=\exp \left(-n \sigma_{m}\right) \sum_{r_{1}=0}^{n} \sum_{r_{2}=0}^{n-r_{1}} \sum_{r_{3}=0}^{n-r_{1}-r_{2}} \ldots \sum_{r_{m}=0}^{n-r_{1}-r_{2}-\cdots-r_{m-1}} \\
\cdot \frac{(-n)_{\delta_{m}} \cdot \prod_{j=1}^{m}\left(-x_{j}\right)_{r_{j}} \cdot \prod_{j=1}^{m}\left(1-e^{\lambda_{j}}\right)^{r_{j}}}{\delta_{m} ! \cdot \prod_{j=1}^{m} r_{j} !} \quad\left(m \in \mathbf{N} ; n \in \mathbf{N}_{0}\right)
\end{gathered}
$$


or, equivalently, by

$$
\begin{gathered}
\varphi_{n}^{m}\left(x_{1}, \ldots, x_{m} ; \lambda_{1}, \ldots, \lambda_{m}\right):=\exp \left(-n \sigma_{m}\right) \\
\cdot \sum_{r_{1}=0}^{n} \sum_{r_{2}=0}^{n-r_{1}} \sum_{r_{3}=0}^{n-r_{1}-r_{2}} \ldots \sum_{r_{m}=0}^{n-r_{1}-r_{2}-\cdots-r_{m-1}}\left(\begin{array}{c}
n \\
\delta_{m}
\end{array}\right)\left(\begin{array}{l}
x_{1} \\
r_{1}
\end{array}\right)\left(\begin{array}{c}
x_{2} \\
r_{2}
\end{array}\right) \cdots\left(\begin{array}{c}
x_{m} \\
r_{m}
\end{array}\right) \\
\cdot \prod_{j=1}^{m}\left(1-e^{\lambda_{j}}\right)^{r_{j}} \quad\left(m \in \mathbf{N} ; n \in \mathbf{N}_{0}\right),
\end{gathered}
$$

where, for convenience,

$$
\sigma_{m}:=\sum_{j=1}^{m} \lambda_{j} \quad \text { and } \quad \delta_{m}:=\sum_{j=1}^{m} r_{j} .
$$

REMARK 1. In its special case when $m=1,(2.1)$ reduces immediately to the second expression for the Gottlieb polynomials $\varphi_{n}(x ; \lambda)$ in (1.1). Moreover, the special cases of $(2.1)$ when $m=2$ and $m=3$ correspond with those investigated in [8] and [9].

In terms of Lauricella's multiple hypergeometric series $F_{D}^{(m)}$ in $m$ variables, defined by (see, for example, [22, p. 33, Eq. 1.4(4)]; see also [11])

$$
\begin{aligned}
& F_{D}^{(m)}[\left.a, b_{1}, \ldots, b_{m} ; c ; z_{1}, \ldots, z_{m}\right] \\
&= \sum_{r_{1}, \ldots, r_{m}=0}^{\infty} \frac{(a)_{r_{1}+\cdots+r_{m}}\left(b_{1}\right)_{r_{1}} \cdots\left(b_{m}\right)_{r_{m}}}{(c)_{r_{1}+\cdots+r_{m}}} \frac{z_{1}^{r_{1}}}{r_{1} !} \cdots \frac{z_{m}^{r_{m}}}{r_{m} !} \\
& \quad\left(\max \left\{\left|z_{1}\right|, \ldots,\left|z_{m}\right|\right\}<1\right),
\end{aligned}
$$

the following generating function holds true for the multivariable and multiparameter Gottlieb polynomials $\varphi_{n}^{m}\left(x_{1}, \ldots, x_{m} ; \lambda_{1}, \ldots, \lambda_{m}\right)$ given by Definition 1 :

$$
\begin{aligned}
& \sum_{n=0}^{\infty} \frac{(\mu)_{n}}{n !} \varphi_{n}^{m}\left(x_{1}, \ldots, x_{m} ; \lambda_{1}, \ldots, \lambda_{m}\right) t^{n}=\left(1-t e^{-\sigma_{m}}\right)^{-\mu} \\
& \quad \cdot F_{D}^{(m)}\left[\mu,-x_{1}, \ldots,-x_{m} ; 1 ; \frac{\left(e^{\lambda_{1}}-1\right) t e^{-\sigma_{m}}}{1-t e^{-\sigma_{m}}}, \ldots, \frac{\left(e^{\lambda_{m}}-1\right) t e^{-\sigma_{m}}}{1-t e^{-\sigma_{m}}}\right] \quad(|t|<1),
\end{aligned}
$$

which, for $m=1$, corresponds obviously to the known generating function for the Gottlieb polynomials $\varphi_{n}(x ; \lambda)$.

With a view to introducing an interesting $q$-extension of the Gottlieb polynomials $\varphi_{n}(x ; \lambda)$ in $(1.1)$, we introduce the following further definitions and notations: 
Generalized q-Binomial Coefficient:

$$
\left[\begin{array}{l}
\lambda \\
n
\end{array}\right]_{q}=\frac{\left(q^{-\lambda} ; q\right)_{n}}{(q ; q)_{n}}\left(-q^{\lambda}\right)^{n} q^{-\left(\begin{array}{c}
n \\
2
\end{array}\right)} \quad\left(\lambda \in \mathbf{C} ; n \in \mathbf{N}_{0}\right) .
$$

q-Exponential Function of the First Kind:

$$
e_{q}(z):=\sum_{k=0}^{\infty} \frac{[(1-q) z]^{k}}{(q ; q)_{k}}=\frac{1}{((1-q) z ; q)_{\infty}}
$$

where we have used the special case of the $q$-binomial theorem (1.16) when $\lambda=0$.

q-Exponential Function of the Second Kind:

$$
E_{q}(z):=\sum_{k=0}^{\infty} q^{\left(\begin{array}{c}
k \\
2
\end{array}\right)} \frac{[(1-q) z]^{k}}{(q ; q)_{k}}
$$

REMARK 2. It is easily seen by applying the definitions (2.7) and (2.8) that

$$
\lim _{q \downarrow 1}\left\{e_{q}(z)\right\}=e^{z}=\lim _{q \downarrow 1}\left\{E_{q}(z)\right\} \quad \text { and } \quad e_{q}(z) \cdot E_{q}(-z)=1 .
$$

Definition 2 (see Khan and Asif [9]). A $q$-extension of the Gottlieb polynomials $\varphi_{n}(x ; \lambda)$ is defined by

$$
\varphi_{n ; q}(x ; \lambda):=\left[E_{q}(-\lambda)\right]^{n} \sum_{k=0}^{n}\left[\begin{array}{l}
n \\
k
\end{array}\right]_{q}\left[\begin{array}{l}
x \\
k
\end{array}\right]_{q} \cdot q^{k(k-1)-x k} \cdot\left[1-e_{q}(\lambda)\right]^{k}
$$

or, equivalently, by

$$
\varphi_{n ; q}(x ; \lambda):=\left[E_{q}(-\lambda)\right]_{2}^{n} \Phi_{1}\left[\begin{array}{c}
q^{-n}, q^{-x} ; \\
q ;
\end{array}\right.
$$

By using some of the $q$-identities presented here, Khan and Asif [9] derived a set of three generating functions for the $q$-Gottlieb polynomials $\varphi_{n ; q}(x ; \lambda)$ given by Definition 2 . We recall the first two of their results in the following corrected forms (cf. [9]):

$$
\sum_{n=0}^{\infty} \varphi_{n ; q}(x ; \lambda) t^{n}=\frac{\left(q t E_{q}(-\lambda) ; q\right)_{\infty}}{\left(q E_{q}(-\lambda) ; q\right)_{\infty}} 1 \Phi_{1}\left[\begin{array}{c}
q^{-x} ; \\
q t E_{q}(-\lambda) ;
\end{array},-\left[1-E_{q}(-\lambda)\right] t\right]
$$

and

$$
\sum_{n=0}^{\infty} \varphi_{n ; q}(x ; \lambda) \frac{t^{n}}{(q ; q)_{n}}=e_{q}\left(\frac{t E_{q}(-\lambda)}{1-q}\right){ }_{1} \Phi_{1}\left[\begin{array}{c}
q^{-x} ; \\
q ;
\end{array},-\left[1-E_{q}(-\lambda)\right] t\right] .
$$


Their third result is given by

$$
\begin{gathered}
\sum_{n=0}^{\infty} \frac{\left(q^{\mu} ; q\right)_{n}}{(q ; q)_{n}} \varphi_{n ; q}(x ; \lambda) t^{n}=\frac{\left(q^{\mu} t E_{q}(-\lambda) ; q\right)_{\infty}}{\left(t E_{q}(-\lambda) ; q\right)_{\infty}} \\
\cdot{ }_{2} \Phi_{2}\left[\begin{array}{c}
q^{\mu}, q^{-x} ; \\
q, q^{\mu} t E_{q}(-\lambda) ;
\end{array} \quad\left[1-E_{q}(-\lambda)\right] t\right] .
\end{gathered}
$$

Motivated essentially by Definition 2, we now define a $q$-extension of the multivariable and multiparameter Gottlieb polynomials $\varphi_{n}^{m}\left(x_{1}, \ldots, x_{m} ; \lambda_{1}, \ldots, \lambda_{m}\right)$ given by (2.2).

DEFINITION 3. A $q$-extension of the generalized (multivariable and multiparameter) Gottlieb polynomials $\varphi_{n}^{m}\left(x_{1}, \ldots, x_{m} ; \lambda_{1}, \ldots, \lambda_{m}\right)$ is defined as follows:

$$
\begin{aligned}
& \varphi_{n ; q}^{m}(\left.x_{1}, \ldots, x_{m} ; \lambda_{1}, \ldots, \lambda_{m}\right):=\left(\prod_{l=1}^{m} E_{q}\left(-\lambda_{l}\right)\right)^{n} \\
& \cdot \sum_{r_{1}=0}^{n} \sum_{r_{2}=0}^{n-r_{1}} \sum_{r_{3}=0}^{n-r_{1}-r_{2}} \cdots \sum_{r_{m}=0}^{n-r_{1}-r_{2}-\cdots-r_{m-1}}\left[\begin{array}{c}
n \\
\delta_{m}
\end{array}\right]_{q} \cdot \prod_{j=1}^{m}\left[\begin{array}{l}
x_{j} \\
r_{j}
\end{array}\right]_{q} \\
& \cdot q^{\sum_{j=1}^{m}\left(\begin{array}{c}
r_{j} \\
2
\end{array}\right)-\sum_{j=1}^{m} x_{j} r_{j}} \cdot \prod_{j=1}^{m}\left[1-e_{q}\left(\lambda_{j}\right)\right]^{r_{j}} \quad\left(m \in \mathbf{N} ; n \in \mathbf{N}_{0}\right),
\end{aligned}
$$

which, by making use of the equations (1.15) and (2.6), can be expressed in the following form:

$$
\begin{aligned}
\varphi_{n ; q}^{m} & \left(x_{1}, \ldots, x_{m} ; \lambda_{1}, \ldots, \lambda_{m}\right)=\left(\prod_{l=1}^{m} E_{q}\left(-\lambda_{l}\right)\right)^{n} \\
\cdot & \sum_{r_{1}=0}^{n} \sum_{r_{2}=0}^{n-r_{1}} \sum_{r_{3}=0}^{n-r_{1}-r_{2}} \ldots \sum_{r_{m}=0}^{n-r_{1}-r_{2}-\cdots-r_{m-1}} q^{-\left(\begin{array}{c}
\delta_{m} \\
2
\end{array}\right)} \frac{\left(q^{-n} ; q\right)_{\delta_{m}}}{(q ; q)_{\delta_{m}}} \\
\cdot & \prod_{j=1}^{m} \frac{\left(q^{\left.-x_{j} ; q\right)_{r_{j}}}\right.}{(q ; q)_{r_{j}}} \cdot \prod_{j=1}^{m}\left\{\left[1-e_{q}\left(\lambda_{j}\right)\right] q^{n}\right\}^{r_{j}} \quad\left(m \in \mathbf{N} ; n \in \mathbf{N}_{0}\right),
\end{aligned}
$$

where, as before, $\delta_{m}$ is given by (2.3).

\section{A Set of $q$-Generating Functions for $\varphi_{n ; q}^{m}\left(x_{1}, \ldots, x_{m} ; \lambda_{1}, \ldots, \lambda_{m}\right)$}

The set of three $q$-generating functions for $\varphi_{n ; q}^{m}\left(x_{1}, \ldots, x_{m} ; \lambda_{1}, \ldots, \lambda_{m}\right)$ (which we state and prove as the main Theorem in this section) are expressed in terms of Srivastava's general basic (or $q$-) hypergeometric series in several variables defined by (see 
[17] and [18]; see also [22, p. 350, Eq. 9.4(284)])

$$
\begin{gathered}
\Phi_{C: D^{\prime} ; \ldots ; D^{(n)}}^{A: B^{\prime} ; \ldots ; B^{(n)}}\left(\begin{array}{c}
{\left[(a): \theta^{\prime}, \ldots, \theta^{(n)}\right]:\left[\left(b^{\prime}\right): \phi^{\prime}\right] ; \ldots ;\left[\left(b^{(n)}\right): \phi^{(n)}\right] ;} \\
{\left[(c): \psi^{\prime}, \ldots, \psi^{(n)}\right]:\left[\left(d^{\prime}\right): \delta^{\prime}\right] ; \ldots ;\left[\left(d^{(n)}\right): \delta^{(n)}\right] ;}
\end{array} ;, z_{1}, \ldots, x_{n}\right) \\
=\sum_{r_{1}, \ldots, r_{n}=0}^{\infty} \frac{\prod_{j=1}^{A}\left(a_{j} ; q\right)_{r_{1} \theta_{j}^{\prime}+\cdots+r_{n} \theta_{j}^{(n)}} \prod_{j=1}^{B^{\prime}}\left(b_{j}^{\prime} ; q\right)_{r_{1} \phi_{j}^{\prime}} \cdots \prod_{j=1}^{B^{(n)}}\left(b_{j}^{(n)} ; q\right)_{r_{n} \phi_{j}^{(n)}}}{\cdot \frac{z_{1}^{r_{1}}}{(q ; q)_{r_{1}}} \cdots \frac{z_{n}^{r_{n}}}{(q ; q)_{r_{n}}},}
\end{gathered}
$$

where the (real or complex) arguments $z_{1}, \ldots, z_{n}$, the complex parameters

$$
\left\{\begin{array}{lll}
a_{j} & (j=1, \ldots, A) ; \quad b_{j}^{(k)} \quad\left(j=1, \ldots, B_{j}^{(k)}\right) ; \\
c_{j} & (j=1, \ldots, C) ; \quad d_{j}^{(k)}\left(j=1, \ldots, D_{j}^{(k)}\right) ; \quad(k=1, \ldots, n)
\end{array}\right.
$$

and the associated coefficients

$$
\left\{\begin{array}{llll}
\theta_{j}^{(k)} & (j=1, \ldots, A) ; & \phi_{j}^{(k)} & \left(j=1, \ldots, B_{j}^{(k)}\right) ; \\
\psi_{j}^{(k)} & (j=1, \ldots, C) ; & \delta_{j}^{(k)} & \left(j=1, \ldots, D_{j}^{(k)}\right) ; \quad(k=1, \ldots, n)
\end{array}\right.
$$

are so constrained that the multiple $q$-series in (3.1) converges.

THEOREM. Each of the following q-generating functions holds true for the multivariable and multiparameter polynomials $\varphi_{n ; q}^{m}\left(x_{1}, \ldots, x_{m} ; \lambda_{1}, \ldots, \lambda_{m}\right)$ defined by (2.14):

$$
\begin{aligned}
& \sum_{n=0}^{\infty} \varphi_{n ; q}^{m}\left(x_{1}, \ldots, x_{m} ; \lambda_{1}, \ldots, \lambda_{m}\right) t^{n}=\frac{\left(q t \prod_{l=1}^{m} E_{q}\left(-\lambda_{l}\right) ; q\right)_{\infty}}{\left(t \prod_{l=1}^{m} E_{q}\left(-\lambda_{l}\right) ; q\right)_{\infty}} \\
& \cdot \Phi_{1: 0 ; \ldots ; 0}^{0: 1 ; \ldots ; 1}\left(\begin{array}{c}
{\left[q^{-x_{1}}: 1\right] ; \ldots ;\left[q^{-x_{m}}: 1\right] ;} \\
{\left[q t \prod_{j=1}^{m} E_{q}\left(-\lambda_{j}\right): 1, \ldots, 1\right]: \ldots ;-}
\end{array} ; \Xi_{1}, \ldots, \Xi_{m}\right),
\end{aligned}
$$

$$
\sum_{n=0}^{\infty} \varphi_{n ; q}^{m}\left(x_{1}, \ldots, x_{m} ; \lambda_{1}, \ldots, \lambda_{m}\right) \frac{t^{n}}{(q ; q)_{n}}=\frac{1}{\left(t \prod_{l=1}^{m} E_{q}\left(-\lambda_{l}\right) ; q\right)_{\infty}}
$$




$$
\cdot \Phi_{1: 0 ; \ldots ; 0}^{0: 1 ; \ldots ;}\left(\begin{array}{l}
{\left[q^{-x_{1}}: 1\right] ; \ldots ;\left[q^{-x_{m}}: 1\right] ;} \\
{[q: 1, \ldots, 1]:} \\
-\ldots ; \Xi_{1}, \ldots, \Xi_{m}
\end{array}\right)
$$

and

$$
\begin{aligned}
& \sum_{n=0}^{\infty} \frac{\left(q^{\mu} ; q\right)_{n}}{(q ; q)_{n}} \varphi_{n ; q}^{m}\left(x_{1}, \ldots, x_{m} ; \lambda_{1}, \ldots, \lambda_{m}\right) t^{n}=\frac{\left(q^{\mu} t \prod_{l=1}^{m} E_{q}\left(-\lambda_{l}\right) ; q\right)_{\infty}}{\left(t \prod_{l=1}^{m} E_{q}\left(-\lambda_{l}\right) ; q\right)_{\infty}} \\
& \cdot \Phi_{2: 0 ; \ldots ; 0}^{1: 1 ; \ldots ; 1}\left(\begin{array}{r}
\left.q^{\mu}: 1, \ldots, 1\right]:\left[q^{-x_{1}}: 1\right] ; \ldots ;\left[q^{-x_{m}}: 1\right] ; \\
{[q: 1, \ldots, 1],\left[q^{\mu} t \prod_{j=1}^{m} E_{q}\left(-\lambda_{j}\right): 1, \ldots, 1\right]:}
\end{array} ; ; \Xi_{1}, \ldots, \Xi_{m}\right),
\end{aligned}
$$

where

$$
\left\{\begin{array}{l}
\Xi_{1}:=\left(\prod_{l=2}^{m} E_{q}\left(-\lambda_{l}\right)\right) \cdot\left[1-E_{q}\left(-\lambda_{1}\right)\right] t \\
\Xi_{2}:=\left(\prod_{\substack{1 \leq l \leq m \\
\overline{(l \neq})}} E_{q}\left(-\lambda_{l}\right)\right) \cdot\left[1-E_{q}\left(-\lambda_{2}\right)\right] t \\
\vdots \\
\Xi_{m}:=\left(\prod_{l=1}^{m-1} E_{q}\left(-\lambda_{l}\right)\right) \cdot\left[1-E_{q}\left(-\lambda_{m}\right)\right] t,
\end{array}\right.
$$

provided that both sides of each of the assertions (3.2), (3.3) and (3.4) exist, it being understood in (3.5) that an empty product is to be interpreted as 1.

PROOF. For convenience, let the left-hand side of the assertion (3.2) be denoted by $\mathcal{S}$. Then, by using the explicit expression for the $q$-polynomials $\varphi_{n ; q}^{m}\left(x_{1}, \ldots, x_{m} ; \lambda_{1}, \ldots, \lambda_{m}\right)$ given by (2.15), we obtain

$$
\begin{aligned}
\mathcal{S}=\sum_{n=0}^{\infty}( & \left.\prod_{l=1}^{m} E_{q}\left(-\lambda_{l}\right)\right)^{n} \cdot \sum_{r_{1}=0}^{n} \sum_{r_{2}=0}^{n-r_{1}} \sum_{r_{3}=0}^{n-r_{1}-r_{2}} \cdots \sum_{r_{m}=0}^{n-r_{1}-r_{2}-\cdots-r_{m-1}} q^{-\left(\begin{array}{c}
\delta_{m} \\
2
\end{array}\right)} \frac{\left(q^{-n} ; q\right)_{\delta_{m}}}{(q ; q)_{\delta_{m}}} \\
& \cdot \prod_{j=1}^{m} \frac{\left(q^{-x_{j}} ; q\right)_{r_{j}}}{(q ; q)_{r_{j}}} \cdot \prod_{j=1}^{m}\left\{q^{n}\left[1-e_{q}\left(\lambda_{j}\right)\right]\right\}^{r_{j}} t^{n},
\end{aligned}
$$

where $\delta_{m}$ and $e_{q}(z)$ are defined by (2.3) and (2.7), respectively.

We now employ the following formal manipulation of double series (see, for example, 
[14, pp. 56-57, Lemma 10] and [23, p. 101, Lemma 1]; see also [2]):

$$
\sum_{n=0}^{\infty} \sum_{l=0}^{n} \Omega(l, n)=\sum_{n=0}^{\infty} \sum_{l=0}^{\infty} \Omega(l, n+l)
$$

and we thus find from (3.6) that

$$
\begin{gathered}
\mathcal{S}=\sum_{n=0}^{\infty} \sum_{r_{1}=0}^{\infty}\left(\prod_{l=1}^{m} E_{q}\left(-\lambda_{l}\right)\right)^{n+r_{1}} \cdot \sum_{r_{2}=0}^{n} \sum_{r_{3}=0}^{n-r_{2}} \cdots \sum_{r_{m}=0}^{n-r_{2}-\cdots-r_{m-1}} q^{-\left(\begin{array}{c}
\delta_{m} \\
2
\end{array}\right)} \frac{\left(q^{-n-r_{1}} ; q\right)_{\delta_{m}}}{(q ; q)_{\delta_{m}}} \\
\cdot \prod_{j=1}^{m} \frac{\left(q^{-x_{j}} ; q\right)_{r_{j}}}{(q ; q)_{r_{j}}} \cdot \prod_{j=1}^{m}\left\{q^{n+r_{1}}\left[1-e_{q}\left(\lambda_{j}\right)\right]\right\}^{r_{j}} t^{n+r_{1}} .
\end{gathered}
$$

By applying the double-series identity (3.7) in (3.8) repeatedly, we get

$$
\begin{array}{r}
\mathcal{S}=\sum_{n=0}^{\infty} \sum_{r_{1}, r_{2}, \ldots, r_{m}=0}^{\infty}\left(\prod_{l=1}^{m} E_{q}\left(-\lambda_{l}\right)\right)^{n+\delta_{m}} \cdot q^{-\left(\begin{array}{c}
\delta_{m} \\
2
\end{array}\right) \cdot \frac{\left(q^{-n-\delta_{m}} ; q\right)_{\delta_{m}}}{(q ; q)_{\delta_{m}}}} \\
\cdot \prod_{j=1}^{m} \frac{\left(q^{-x_{j}} ; q\right)_{r_{j}}}{(q ; q)_{r_{j}}} \cdot \prod_{j=1}^{m}\left\{q^{n+\delta_{m}}\left[1-e_{q}\left(\lambda_{j}\right)\right]\right\}^{r_{j}} \cdot t^{n+\delta_{m}}
\end{array}
$$

In view of the $q$-identity (1.15) and the following elementary formula:

$$
(\lambda ; q)_{n+k}=(\lambda ; q)_{n}\left(\lambda q^{n} ; q\right)_{k} \quad\left(n, k \in \mathbf{N}_{0} ; \lambda \in \mathbf{C}\right),
$$

it is easily seen that

$$
\begin{aligned}
\left(q^{-n-\delta_{m}} ; q\right)_{\delta_{m}} & =(-1)^{\delta_{m}} q^{\left(\begin{array}{c}
\delta_{m} \\
2
\end{array}\right)-\left(n+\delta_{m}\right) \delta_{m}} \frac{(q ; q)_{n+\delta_{m}}}{(q ; q)_{n}} \\
& =(-1)^{\delta_{m}} q^{\left(\begin{array}{c}
\delta_{m} \\
2
\end{array}\right)-\left(n+\delta_{m}\right) \delta_{m}} \frac{(q ; q)_{\delta_{m}}\left(q^{\delta_{m}+1} ; q\right)_{n}}{(q ; q)_{n}}
\end{aligned}
$$

Upon applying this last formula (3.11) to (3.9), if we rearrange the resulting multiple series, we obtain

$$
\begin{gathered}
\mathcal{S}=\sum_{r_{1}, \ldots, r_{m}=0}^{\infty} \prod_{j=1}^{m}\left(t\left[e_{q}\left(\lambda_{j}\right)-1\right] \cdot \prod_{l=1}^{m} E_{q}\left(-\lambda_{l}\right)\right)^{r_{j}} \cdot \prod_{j=1}^{m} \frac{\left(q^{-x_{j}} ; q\right)_{r_{j}}}{(q ; q)_{r_{j}}} \\
\cdot \sum_{n=0}^{\infty} \frac{\left(q^{\delta_{m}+1} ; q\right)_{n}}{(q ; q)_{n}}\left(t \prod_{j=1}^{m} E_{q}\left(-\lambda_{j}\right)\right)^{n},
\end{gathered}
$$

which, in light of the $q$-binomial theorem (1.16) and the $q$-identity (1.10), yields

$$
\sum_{n=0}^{\infty} \frac{\left(q^{\delta_{m}+1} ; q\right)_{n}}{(q ; q)_{n}}\left(t \prod_{j=1}^{m} E_{q}\left(-\lambda_{j}\right)\right)^{n}
$$




$$
\begin{aligned}
& =\frac{\left(q^{\delta_{m}+1} t \prod_{j=1}^{m} E_{q}\left(-\lambda_{j}\right) ; q\right)_{\infty}}{\left(t \prod_{j=1}^{m} E_{q}\left(-\lambda_{j}\right) ; q\right)_{\infty}} \\
& =\frac{\left(q t \prod_{j=1}^{m} E_{q}\left(-\lambda_{j}\right) ; q\right)_{\infty}}{\left(q t \prod_{j=1}^{m} E_{q}\left(-\lambda_{j}\right) ; q\right)_{\delta_{m}} \cdot\left(t \prod_{j=1}^{m} E_{q}\left(-\lambda_{j}\right) ; q\right)_{\infty}} .
\end{aligned}
$$

If we now substitute from (3.13) for the last summation in (3.12) and use the second identity under Remark 2, we find that

$$
\begin{gathered}
\mathcal{S}=\frac{\left(q t \prod_{j=1}^{m} E_{q}\left(-\lambda_{j}\right) ; q\right)_{\infty}}{\left(t \prod_{j=1}^{m} E_{q}\left(-\lambda_{j}\right) ; q\right)_{\infty}} \sum_{r_{1}, \ldots, r_{m}=0}^{\infty} \frac{1}{\left(q t \prod_{j=1}^{m} E_{q}\left(-\lambda_{j}\right) ; q\right)_{\delta_{m}}} \\
\cdot \prod_{j=1}^{m}\left(q^{-x_{j}} ; q\right)_{r_{j}} \cdot \prod_{j=1}^{m} \frac{\left(t\left[1-E_{q}\left(-\lambda_{j}\right)\right] \cdot \prod_{\substack{1 \leq l \leq m \\
(l \neq j)}} E_{q}\left(-\lambda_{l}\right)\right)^{r_{j}}}{(q ; q)_{r_{j}}},
\end{gathered}
$$

where $\delta_{m}$ is defined (as before) by (2.3).

Finally, we interpret the multiple $q$-series in (3.14) as a special case of Srivastava's general basic (or $q$-) hypergeometric series in several variables defined by (3.1). We are thus led to the first assertion (3.2) of the Theorem. The other two assertions (3.3) and (3.4) of the Theorem can be proved by a similar argument.

Alternatively, with a view to providing a relatively shorter demonstration of (for example) the assertion (3.4) of the Theorem, we choose to rewrite the definition (2.15) as follows:

$$
\begin{gathered}
\varphi_{n ; q}^{m}\left(x_{1}, \ldots, x_{m} ; \lambda_{1}, \ldots, \lambda_{m}\right)=\left(\prod_{l=1}^{m} E_{q}\left(-\lambda_{l}\right)\right)^{n} \sum_{r_{1}, \ldots, r_{m}=0}^{r_{1}+\cdots+r_{m} \leqq n} q^{n \delta_{m}-\left(\begin{array}{c}
\delta_{m} \\
2
\end{array}\right)} \frac{\left(q^{-n} ; q\right)_{\delta_{m}}}{(q ; q)_{\delta_{m}}} \\
\cdot \prod_{j=1}^{m} \frac{\left(q^{-x_{j}} ; q\right)_{r_{j}}}{(q ; q)_{r_{j}}} \cdot \prod_{j=1}^{m}\left[1-e_{q}\left(\lambda_{j}\right)\right]^{r_{j}} \quad\left(m \in \mathbf{N} ; n \in \mathbf{N}_{0}\right),
\end{gathered}
$$

where, as before, $\delta_{m}$ is given by (2.3). We now denote the first member of the assertion (3.4) of the Theorem by $\mathfrak{S}_{\mu}$. Then, by appealing appropriately to a known multiple-series extension 
of the series identity (3.7) (see, for example, [23, p. 102, Lemma 4]), we find that

$$
\begin{gathered}
\mathfrak{S}_{\mu}=\sum_{r_{1}, \ldots, r_{m}=0}^{\infty} \frac{\left(q^{\mu} ; q\right)_{\delta_{m}}}{(q ; q)_{\delta_{m}}} \prod_{j=1}^{m}\left(t\left[e_{q}\left(\lambda_{j}\right)-1\right] \cdot \prod_{l=1}^{m} E_{q}\left(-\lambda_{l}\right)\right)^{r_{j}} \cdot \prod_{j=1}^{m} \frac{\left(q^{-x_{j}} ; q\right)_{r_{j}}}{(q ; q)_{r_{j}}} \\
\cdot \sum_{n=0}^{\infty} \frac{\left(q^{\mu+\delta_{m}} ; q\right)_{n}}{(q ; q)_{n}}\left(t \prod_{j=1}^{m} E_{q}\left(-\lambda_{j}\right)\right)^{n} \cdot
\end{gathered}
$$

By using the $q$-binomial theorem (1.16), the $q$-identity (1.10) and the second identity under Remark 2, we are led eventually to the following expression for $\mathfrak{S}_{\mu}$ :

$$
\begin{gathered}
\mathfrak{S}_{\mu}=\frac{\left(q^{\mu} t \prod_{j=1}^{m} E_{q}\left(-\lambda_{j}\right) ; q\right)_{\infty}}{\left(t \prod_{j=1}^{m} E_{q}\left(-\lambda_{j}\right) ; q\right)_{\infty}} \sum_{r_{1}, \ldots, r_{m}=0}^{\infty} \frac{\left(q^{\mu} ; q\right)_{\delta_{m}}}{(q ; q)_{\delta_{m}}\left(q^{\mu} t \prod_{j=1}^{m} E_{q}\left(-\lambda_{j}\right) ; q\right)_{\delta_{m}}} \\
\cdot \prod_{j=1}^{m}\left(q^{-x_{j}} ; q\right)_{r_{j}} \cdot \prod_{j=1}^{m} \frac{\left(t\left[1-E_{q}\left(-\lambda_{j}\right)\right] \cdot \prod_{\substack{1 \leq l \leq m \\
(l \neq j)}} E_{q}\left(-\lambda_{l}\right)\right)^{r_{j}}}{(q ; q)_{r_{j}}},
\end{gathered}
$$

which, when interpreted as a special case of Srivastava's general basic (or q-) hypergeometric series in several variables defined by (3.1), yields the second member of the assertion (3.4) of the Theorem. Thus, evidently, we have completed the proof of the Theorem.

REMARK 3. The special cases of the assertions (3.2), (3.3) and (3.4) of the Theorem would correspond to the $q$-generating functions (2.11), (2.12) and (2.13), respectively. For many other interesting families of $q$-generating functions in one, two and more variables, the reader may be referred (for example) to the earlier work by Srivastava [19].

An Open Problem. By deriving a multivariable and multiparameter analogue of the generating function (1.6) for the $q$-Gottlieb polynomials $\varphi_{n ; q}^{m}\left(x_{1}, \ldots, x_{m} ; \lambda_{1}, \ldots, \lambda_{m}\right)$ defined by (2.14), it would seem to be worthwhile to develop various families of bilinear, bilateral or mixed multilateral $q$-generating functions by following Srivastava's theory based upon the following general family of generating functions (see, for example, [15], [16] and [23, p. 438, Eq. 8.5(1)]):

$$
\begin{aligned}
\sum_{n=0}^{\infty} \gamma_{\mu, n} \Delta\left(x_{1}, \ldots, x_{m}\right) t^{n}=\frac{\theta\left(x_{1}, \ldots, x_{m} ; t\right)}{\left[\phi\left(x_{1}, \ldots, x_{m} ; t\right)\right]^{\mu}} \\
\cdot \Delta_{\mu}\left(\psi_{1}\left(x_{1}, \ldots, x_{m} ; t\right), \ldots, \psi_{m}\left(x_{1}, \ldots, x_{m} ; t\right)\right),
\end{aligned}
$$


where $\mu$ is an arbitrary complex number, the coefficients $\gamma_{\mu, n}\left(n \in \mathbf{N}_{0}\right)$ are suitable constants and $\theta, \phi, \psi_{1}, \ldots, \psi_{m}$ are suitable functions of $x_{1}, \ldots, x_{m}$ and $t$.

ACKNOWLEDGEMEnts. This paper was supported by the Basic Science Research Program through the National Research Foundation of the Republic of Korea funded by the Ministry of Education, Science and Technology (Grant No. 2012-0002957). The present investigation was supported, in part, by the Natural Sciences and Engineering Research Council of Canada under Grant OGP0007353.

\section{References}

[1] J. CAO and H. M. SRivastava, Some $q$-generating functions of the Carlitz and Srivastava-Agarwal types associated with the generalized Hahn polynomials and the generalized Rogers-Szegö polynomials, Appl. Math. Comput. 219 (2013), 8398-8406.

[2] J. CHOI, Notes on formal manipulations of double series, Commun. Korean Math. Soc. 18 (2003), 781-789.

[ 3 ] J. CHOI, A generalization of Gottlieb polynomials in several variables, Appl. Math. Lett. 25 (2012), 43-46.

[ 4 ] J. CHOI, $q$-Extension of a generalization of Gottlieb polynomials in two variables, J. Chungcheong Math. Soc. 25 (2012), 253-265.

[ 5 ] J. CHOI, $q$-Extension of a generalization of Gottlieb polynomials in three variables, Honam Math. J. 34 (2012), 327-340.

[ 6 ] G. Gasper and M. Rahman, Basic Hypergeometric Series (with a Foreword by Richard Askey), Encyclopedia of Mathematics and Its Applications, Vol. 35, Cambridge University Press, Cambridge, New York, Port Chester, Melbourne and Sydney, 1990; Second edition, Encyclopedia of Mathematics and Its Applications, Vol. 96, Cambridge University Press, Cambridge, London and New York, 2004.

[ 7 ] M. J. GotTlieb, Concerning some polynomials orthogonal on a finite or enumerable set of points, Amer. J. Math. 60(2) (1938), 453-458.

[ 8 ] M. A. KHAN and M. AKHLAQ, Some new generating functions for Gottlieb polynomials of several variables, Internat. Trans. Appl. Sci. 1 (2009), 567-570.

[9] M. A. KHAN and M. AsiF, A note on generating functions of $q$-Gottlieb polynomials, Commun. Korean Math. Soc. 27 (2012), 159-166.

[10] M. A. Khan, A. H. KHAN and M. Singh, Integral representations for the product of Krawtchouk, Meixner, Charlier and Gottlieb polynomials, Internat. J. Math. Anal. (Ruse) 5 (2011), 199-206.

[11] G. Lauricella, Sulle funzioni ipergeometriche a più variabili, Rend. Circ. Mat. Palermo 7 (1893), 111-158.

[12] E. B. MCBRIDE, Obtaining Generating Functions, Springer Tracts in Natural Philosophy, Vol. 21, SpringerVerlag, New York, Heidelberg and Berlin, 1971.

[13] J. MeIXnER, Orthogonale Polynomsysteme mit einer besonderen Gestalt der erzeugenden Funktion, J. London Math. Soc. 9 (1934), 6-13.

[14] E. D. Rainville, Special Functions, Macmillan Company, New York, 1960; Reprinted by Chelsea Publishing Company, Bronx, New York, 1971.

[15] H. M. SRIVASTAVA, Some bilateral generating functions for a certain class of special functions. I, Nederl. Akad. Wetensch. Proc. Ser. A 83 = Indag. Math. 42 (1980), 221-233.

[16] H. M. SRivastava, Some bilateral generating functions for a certain class of special functions. II, Nederl. Akad. Wetensch. Proc. Ser. A 83 = Indag. Math. 42 (1980), 234-246.

[17] H. M. SRIvastava, Certain $q$-polynomial expansions for functions of several variables, IMA J. Appl. Math. 30 (1983), 315-323.

[18] H. M. SRivastava, Certain $q$-polynomial expansions for functions of several variables. II, IMA J. Appl. Math. 33 (1984), 205-209. 
[19] H. M. SRivastava, A family of $q$-generating functions, Bull. Inst. Math. Acad. Sinica 12 (1984), 327-336.

[20] H. M. SRIVAstaVA, Some generalizations and basic (or $q$-) extensions of the Bernoulli, Euler and Genocchi polynomials, Appl. Math. Inform. Sci. 5 (2011), 390-444.

[21] H. M. SRivastaVA and J. CHOI, Zeta and q-Zeta Functions and Associated Series and Integrals, Elsevier Science Publishers, Amsterdam, London and New York, 2012.

[22] H. M. SRivastava and P. W. Karlsson, Multiple Gaussian Hypergeometric Series, Halsted Press (Ellis Horwood Limited, Chichester), John Wiley and Sons, New York, Chichester, Brisbane and Toronto, 1985.

[23] H. M. SRivastava and H. L. Manocha, A Treatise on Generating Functions, Halsted Press (Ellis Horwood Limited, Chichester), John Wiley and Sons, New York, Chichester, Brisbane and Toronto, 1984.

[24] G. SzEgö, Orthogonal Polynomials, Fourth edition, American Mathematical Society Colloquium Publications, Vol. 23, American Mathematical Society, Providence, Rhode Island, 1975.

Present Addresses:

JUNESANG CHOI

Department of Mathematics,

DONGGUK UNIVERSITY,

GYEONGJU 780-714, REPUBLIC OF KOREA.

e-mail: junesang@mail.dongguk.ac.kr

H. M. SRIVASTAVA

DEPARTMENT OF MATHEMATICS AND STATISTICS,

UNIVERSITY OF VICTORIA,

Victoria, British Columbia V8W 3R4, CANAda.

e-mail: harimsri@math.uvic.ca 